\title{
LA INSUFICIENTE REGULACIÓN DE LOS ACUERDOS PESQUEROS DURMIENTES EN EL REGLAMENTO (UE) $2017 / 2403$ SOBRE LA GESTIÓN SOSTENIBLE DE LAS FLOTAS EXTERIORES
}

\author{
CARLOS TEIJO GARCÍA ${ }^{1}$ \\ Universidad de Santiago de Compostela \\ carlos.teijo@usc.es
}

\section{Resumen}

El presente trabajo aborda un problema jurídico relevante pero poco estudiado - los efectos de la cláusula de exclusividad sobre los denominados acuerdos durmientes- que se genera en la práctica relativa a la ejecución de los tratados de carácter pesquero suscritos por la UE con terceros Estados. El artículo analiza la situación que se

1 Profesor contratado doctor de Derecho Internacional Público y Relaciones Internacionales de la Universidad de Santiago de Compostela. Investigación realizada en el marco del Proyecto «La reforma de la gobernanza pesquera internacional y europea. Retos para el sector pesquero español» (DER2013-45923-R), financiado por el Ministerio de Economía y Competitividad. El autor agradece las observaciones realizadas por los dos evaluadores anónimos del trabajo. 
plantea cuando un acuerdo pesquero carece durante años de protocolo de ejecución operativo pero, en virtud de la cláusula de exclusividad del tratado que continúa en vigor, la flota exterior de la UE no puede acceder a los caladeros del Estado ribereño mediante otro tipo de solución jurídica como pudieran ser las licencias privadas o los contratos de chárter. El artículo revisa el alcance e implicaciones de este problema y valora la respuesta que da a la cuestión el nuevo Reglamento (UE) 2017/2403.

\section{Palabras clave}

Unión Europea; Política Pesquera Común; acuerdos de pesca; cláusula de exclusividad.

\section{THE POOR TREATMENT OF DORMANT FISHERIES AGREEMENTS IN REGULATION (EU) 2017/2403 ON THE SUSTAINABLE MANAGEMENT OF EXTERNAL FISHING FLEETS}

\section{Abstract}

This article analyses the harmful effects of the exclusivity clause on so-called dormant agreements, a relevant but unstudied legal problem that arises in the EU's fisheries treaty practice with third States. The paper focuses on the situation that appears when a fisheries agreement lacks an operational execution protocol for years but, because of the exclusivity clause of the treaty that continues in force, the EU external fleet cannot access the coastal third State's fisheries resources through another kind of legal arrangement such as a private license or a charter contract. The article reviews the scope and implications of this problem and criticices the unsatisfactory treatment of this issue included in new Regulation (EU) 2017/2403.

\section{Keywords} clause.

European Union; Common Fisheries Policy; fisheries agreements; exclusivity

\section{LA INSUFFISANTE REGLEMENTATION DES ACCORDS DE PECHE DORMANTS DANS LE REGLEMENT (UE) 2017/2403 RELATIF Á LA GESTION DURABLE DES FLOTTES DE PECHE EXTERNES}

\section{Résumé}

Le présent travail aborde un problème juridique important mais peu étudié -les négatifs effets de la clause d'exclusivité sur les accords dormants- qui est survenu dans la pratique relatif à l'exécution des traités de pêche signés par l'UE 
avec des États tiers. L'article analyse la situation qui se présente lorsqu'un accord de pêche manque longtemps d'un protocole d'exécution opérationnel mais, en vertu de la clause d'exclusivité du traité qui reste en vigueur, la flotte externe de l'UE ne peut y accéder aux zones de pêche de l'État côtier à travers un autre type de solution juridique, comme une licence privée ou un contrat d'affrètement. L'article passe en revue la portée et les implications de ce problème juridique et critique la résponse que el nouveau Règlement (UE) 2017/2403 donne à la question.

\section{Mots clés}

Union Européenne; Politique Commun de Pêche; accords de pêche; clause d'exclusivité. 


\section{SUMARIO}

I. PLANTEAMIENTO Y CONTEXTUALIZACIÓN: LAS MODALIDADES DE ACCESO DE LA FLOTA PESQUERA DE ALTURA COMUNITARIA A LAS AGUAS DE UN TERCER ESTADO: 1. La vía principal de acceso: estructura y funciones de los Acuerdos de Colaboración para la Pesca Sostenible (ACPS). 2. Las fórmulas complementarias: licencias de carácter privado y acuerdos de flete. II. LA INCLUSIÓN DE CLÁUSULAS DE EXCLUSIVIDAD EN LOS ACPS. III. EL PERNICIOSO EFECTO COLATERAL DE LAS CLÁUSULAS DE EXCLUSIVIDAD SOBRE LOS ACUERDOS DURMIENTES. IV. UNA VENTANA DE OPORTUNIDAD PARA RESOLVER LA PROBLEMÁTICA VINCULADA A LOS ACUERDOS DURMIENTES: EL PROCEDIMIENTO LEGISLATIVO DE ADOPCIÓN DEL REGLAMENTO (UE) 2017/2403 SOBRE LA GESTIÓN SOSTENIBLE DE LAS FLOTAS PESQUERAS EXTERIORES. V. CONCLUSIONES. BIBLIOGRAFÍA.

\section{PLANTEAMIENTO Y CONTEXTUALIZACIÓN: LAS MODALIDADES DE ACCESO DE LA FLOTA PESQUERA DE ALTURA COMUNITARIA A LAS AGUAS DE UN TERCER ESTADO}

La flota de altura y gran altura europea que pesca en aguas extracomunitarias tiene una notable relevancia en términos económicos y sociales. Aunque el segmento de aguas distantes constituye menos del $1 \%$ de la flota de la UE, con apenas 285 de los 84420 buques registrados, aporta el $14 \%$ de las capturas totales y el $19 \%$ del tonelaje bruto total (STECF, 2017: 63) ${ }^{2}$. En el caso de

2 Se encuentran incluidos dentro de la flota de aguas distantes los navíos que cumplen los parámetros de poseer una eslora superior a los 24 metros y operar en aguas extracomunitarias, incluyendo las regiones pesqueras más alejadas de la UE. La delimitación de los perfiles de la flota de aguas distantes no resulta, sin embargo, pacífica. En 2015, el proyecto Who Fishes Far (http://whofishesfar.org) —auspiciado por varias ONG medioambientalistas - publicó una base de datos con información relativa a los buques de bandera de Estados miembros de la UE que habían recibido autorizaciones para pescar en aguas no comunitarias. Los registros de dicha base señalan que, entre 2008 y 2015, 23239 navíos fueron autorizados para realizar actividades pesqueras más allá de las aguas comunitarias por lo que, en apariencia (tal y como da a entender la propia web), la flota de aguas distantes es mayor de lo que admiten los 
España, que es el principal Estado europeo de pesca a distancia, la relevancia de la actividad de los buques de altura y gran altura es incluso superior puesto que en 2016 integraban esta flota tan solo 194 navíos —entre los 9299 pesqueros existentes en España—, si bien dichos buques, que faenan en alta mar y en las Zonas Económicas Exclusivas (ZEE) de terceros países, generaban el $56 \%$ de la captura total (MAGRAMA, 2017; CEPESCA, 2017: 9).

Cuando esta flota faena en aguas extracomunitarias puede hacerlo al amparo de cinco modalidades básicas de cobertura jurídica: con licencia para pescar en aguas internacionales gestionadas por una Organización Regional de Ordenación Pesquera (OROP); en un área de alta mar que no se encuentre bajo el mandato de una OROP; en la ZEE de un tercer Estado bajo el paraguas de un Acuerdo de Colaboración para la Pesca Sostenible (ACPS) suscrito por la UE con dicho país ribereño; en la ZEE de un tercer Estado al amparo de una licencia de carácter privado expedida directamente por las autoridades del ribereño en favor de los armadores comunitarios; o, por último, mediante la suscripción de contratos de flete con armadores de terceros países (habitualmente nacionales del Estado ribereño) mediante los que los buques de bandera europea son alquilados para faenar con una licencia no comunitaria. Nuestro análisis va a detenerse sobre la interacción problemática que se establece entre las tres últimas modalidades de acceso a la ZEE de un tercer Estado cuando se da la circunstancia de que la UE mantiene vigente con este un convenio de pesca que incluye una cláusula de exclusividad que, sin embargo, carece de protocolo de aplicación.

registros oficiales de la Comisión Europea. Ciertamente, una parte importante de los buques incluidos en el recuento oficioso realizado por las ONG y que han obtenido autorización para la pesca en aguas extracomunitarias lo han hecho durante períodos de tiempo muy limitados y con el objetivo de beneficiarse de los acuerdos de reciprocidad suscritos con Noruega, Islandia y las islas Feroe en aguas colindantes con las comunitarias, así como para operar en el Mediterráneo y el mar Negro bajo los auspicios de las OROP regionales competentes, esto es, la Comisión Internacional para la Conservación del Atún Atlántico (ICCAT) y la Comisión General de Pesca del Mediterráneo (GFCM). Teniendo en cuenta esta circunstancia, resulta exagerado integrar a la totalidad de dichos buques — que operan solo de manera esporádica fuera de aguas comunitarias - dentro del segmento de la flota de aguas distantes. En cualquier caso y teniendo en cuenta el debate existente sobre la cuestión, conviene precisar, en aras de la claridad analítica, que los datos empleados por este estudio para dimensionar el tamaño de la flota comunitaria de aguas distantes son los oficiales y disponibles que proporciona el Comité Científico, Técnico y Económico de Pesca de la UE (creado mediante Decisión de la Comisión de 25 de febrero de 2016, DO C 74, 26.2.2016) que cifra el número de navíos que la conforman, en 2017, en 285. 


\section{LA VÍA PRINCIPAL DE ACCESO: ESTRUCTURA Y FUNCIONES DE LOS ACUERDOS DE COLABORACIÓN PARA LA PESCA SOSTENIBLE (ACPS)}

Los ACPS son tratados internacionales que la UE celebra con terceros Estados con el fin de obtener el acceso a sus recursos o a sus aguas para explotar de forma sostenible una parte del excedente de recursos biológicos marinos, a cambio de una compensación financiera que puede incluir apoyo sectorial $^{3}$. La UE posee competencia exclusiva para la celebración de acuerdos internacionales de acceso a recursos pesqueros (art. 4 TFUE). En ejercicio de esta atribución ha suscrito dos grandes conjuntos de acuerdos, en primer término, los denominados septentrionales con países y territorios del norte de Europa (como Noruega, Islandia y las islas Feroe) cuya estructura se basa en convenios de reciprocidad orientados hacia la gestión conjunta de recursos compartidos. Y, por otra parte, los denominados en la actualidad Acuerdos de Cooperación para la Pesca Sostenible (ACPS) a través de los que la UE ofrece una contrapartida financiera y técnica a cambio de derechos de pesca a países asociados del sur ${ }^{4}$; a su vez, los ACPS se dividen en dos subcategorías como son los acuerdos atuneros, que permiten a los buques de la flota comunitaria perseguir a las poblaciones migratorias de túnidos en sus desplazamientos por las aguas de África, el océano Índico y el Pacífico, y los acuerdos de carácter mixto o multiespecie, que conceden acceso a diversas poblaciones de peces en la ZEE del país contraparte (sobre la reciente práctica convencional comunitaria en materia de pesca, véanse Sobrino y Oanta, 2015; Teijo García, 2016).

La estructura de los acuerdos pivota sobre la contrapartida financiera que abona la UE a los países asociados a cambio del acceso de la flota. En esta contraprestación resulta posible distinguir un primer elemento, ligado a la obtención de derechos de acceso a la ZEE del Estado ribereño, y una segunda partida, vinculada al apoyo financiero «sectorial» para el fomento de la pesca sostenible del país asociado, que pretende reforzar la gestión, supervisión y la vigilancia de la actividad extractiva. El esquema de la relación asociativa a largo plazo entre la UE y el Estado contraparte se establece en el ACPS, si bien las condiciones concretas en las que se realizará la actividad pesquera vienen

3 Art. 4.1.37 del Reglamento (UE) 1380/2013 del Parlamento Europeo y del Consejo, de 11-12-2013, sobre la política pesquera común, por el que se modifican los Reglamentos (CE) 1954/2003 y (CE) 1224/2009 del Consejo, y se derogan los Reglamentos (CE) 2371/2002 y (CE) 639/2004 del Consejo y la Decisión 2004/585/CE del Consejo, DO L 354, 28-12-2013.

4 El Reglamento de base de la PPC rebautiza como Acuerdos de Cooperación para la Pesca Sostenible a los que habían sido denominados, desde 2004, Acuerdos para la Asociación Pesquera, véanse los arts. 31-32 del Reglamento (UE) 1380/2013. 
determinadas por lo previsto en los protocolos de aplicación, que acostumbran a tener una vigencia limitada que bascula entre tres y seis años.

A efectos contextuales para nuestro análisis, es preciso indicar que la UE mantiene vigentes (en noviembre de 2017) los protocolos de doce ACPS con Groenlandia (hasta el 31-12-2020), Marruecos (hasta el 14-07-2018), Mauritania (hasta el 15-11-2019, aplicación provisional), Senegal (hasta el 19-11-2019), Cabo Verde (hasta el 22-12-2018), Guinea-Bisáu (hasta el 2311-17), Liberia (hasta el 8-12-2020, aplicación provisional), Costa de Marfil (hasta el 30-06-2018), Sao Tome e Príncipe (hasta el 22-05-2018), Madagascar (hasta 31-12-2018), Seychelles (hasta el 17-07-2020) e islas Cook (hasta 24-10-2019, aplicación provisional); estos convenios han sido suscritos en su mayoría para la pesca del atún, con la excepción de los suscritos con Mauritania, Marruecos, Guinea-Bisáu y Groenlandia. Con leves fluctuaciones, derivadas de la pérdida de vigencia de alguno de los protocolos, el mantenimiento de los acuerdos de pesca ha supuesto durante el último lustro un coste aproximado de 145 millones de euros al año (el $13 \%$ del presupuesto de la Política Pesquera Común, PPC), que ha sido destinado mayoritariamente a la financiación de los acuerdos de carácter mixto suscritos con Mauritania y Marruecos (Popescu, 2015: 1).

La estrategia de futuro de la política convencional pesquera ejecutada por la Comisión Europea pasa, en principio, por ampliar la red de acuerdos existente, en consonancia con las prioridades del sector. Los armadores han expresado un interés prioritario por la firma de tratados con países como Angola, Sierra Leona, Guinea-Conakri, Tanzania y Kenia así como por la reactivación del protocolo con Mozambique. Al tener la mayoría de los ACPS en vigor carácter atunero, la estrategia negociadora de la Comisión se orienta a favorecer la continuidad de la actividad extractiva de la flota a lo largo de las diversas ZEE de los Estados ribereños del área regional en cuestión, sea África occidental o el Índico, debido la naturaleza transzonal y altamente migratoria de las poblaciones de túnidos (Popescu, 2016). En la práctica convencional de los últimos años podemos observar tanto una creciente dificultad para la suscripción de acuerdos multiespecie como una tendencia correlativa al incremento de la celebración de convenios atuneros. Desde esa perspectiva, la posición relativa de la flota comunitaria demersal resulta más frágil en el contexto de la dimensión externa de la PPC puesto que sus prácticas de explotación de recursos presentan una mayor probabilidad de entrar en conflicto con la flota artesanal local y porque el excedente pesquero sobre el que ha incidido tradicionalmente su actividad muestra niveles decrecientes, como ocurre en los casos de Costa de Marfil, Mozambique, Gabón y Senegal, donde los acuerdos mixtos han sido transformados en atuneros (Mulazzani y Malorgio, 2015: 8). 
Desde el punto de vista socioeconómico, los acuerdos de pesca con terceros países suponen en torno al $8 \%$ de las capturas totales de la flota comunitaria, constituyen una fuente relevante de suministro para la industria transformadora y generan un importante volumen de empleo puesto que 35000 puestos de trabajo directos dependen de estos convenios. Por otro lado, los acuerdos también resultan económicamente capitales para los Estados contraparte al constituir una fuente sustanciosa de ingresos. En algunos casos la dependencia financiera respecto a los ingresos pesqueros alcanza niveles sorprendentes como ocurre con Mauritania y Guinea-Bisáu, donde estos recursos financieros han llegado a suponer el 20 y el $38 \%$, respectivamente, de los ingresos anuales del Estado (Walmsley et al., 2007: 60; Hudson, 2007: 1).

\section{LAS FÓRMULAS COMPLEMENTARIAS: LICENCIAS DE CARÁCTER PRIVADO Y ACUERDOS DE FLETE}

El acceso de la flota comunitaria a las ZEE de los países terceros con los que la UE no ha suscrito un acuerdo de pesca ha de realizarse mediante otras soluciones alternativas como la expedición por parte del Estado ribereño de licencias privadas de pesca. Sobre esta modalidad de acceso disponemos de una cantidad mucho menor de información puesto que, a diferencia de lo que ocurre con los ACPS, la Comisión Europea carece de una base de datos exhaustiva que nos permita delimitar los perfiles de esta práctica. De forma aproximada, la Comisión ha calculado que cada año se expiden entre 150 y 180 licencias privadas de pesca que se concentran, sobre todo, en zonas con una elevada presión sobre los recursos ictícolas como el golfo de Guinea, Angola, Namibia y el noroeste del océano Índico5.

Hasta la reciente entrada en vigor (17-1-2018) del Reglamento (UE) 2017/2403 sobre la gestión sostenible de las flotas pesqueras ${ }^{6}$, los Estados miembros de la UE cuyos buques participaban en actividades extractivas en las aguas de países terceros mediante acuerdos de carácter privado estaban obligados únicamente a comunicar a la Comisión Europea el nombre de los

5 Comisión Europea, Analyse d'impact accompagnant le document: Proposition de Règlement du Parlement Européen et du Conseil relatif à la gestion durable des flottes de pêche externes, abrogeant le Règlement (CE) 1006/2008 du Conseil, 10-12-2015, SWD(2015) 279 final, p. 15.

6 Reglamento (UE) 2017/2403 del Parlamento Europeo y del Consejo de 12 de diciembre de 2017, sobre la gestión sostenible de las flotas pesqueras exteriores y por el que se deroga el Reglamento (CE) 1006/2008 del Consejo, DO L 347, 28-12-2017. 
navíos implicados ${ }^{7}$, sin que debiesen proporcionar otros datos relevantes como puede ser la especie objetivo, la zona de pesca, el período o las artes de pesca utilizadas. La Comisión ha carecido tradicionalmente, por tanto, de capacidad para proporcionar información sobre el alcance de las actividades que realizan estos buques y tampoco ha tenido competencia para verificar la conformidad de los acuerdos con la legislación pesquera y laboral de la UE.

La necesidad de incrementar el nivel de transparencia de la red de acuerdos privados suscritos por los armadores europeos con terceros Estados ha sido una reivindicación constante a lo largo de los últimos años ${ }^{8}$, si bien los datos disponibles continúan siendo escasos y fragmentarios. Las peticiones formales de información realizadas por varias ONG a los Estados comunitarios de pabellón sobre este particular, entre mayo de 2015 y septiembre de 2016, se han saldado con magros resultados puesto que Croacia, Finlandia, Francia, Grecia, Italia, Malta, Portugal y Rumanía ni siquiera han respondido al requerimiento; Holanda ha indicado que no podía proporcionar dichos datos, al tener carácter privado; Alemania, Bélgica, Bulgaria, Chipre, Dinamarca, Eslovaquia, Irlanda, Letonia, Polonia y Reino Unido señalaron que no habían autorizado la expedición de licencias privadas; y, por último, Estonia, Lituania y España suministraron información muy limitada (que en el caso español ni siquiera fue hecha pública en el informe no gubernamental) sobre un pequeño contingente de licencias para faenar en Angola, Guinea-Conakri y Senegal (EJF et al., 2016: 6).

Por otra parte, la información pública a la que resulta posible acceder sobre los acuerdos privados resulta poco sistemática y aparece deslavazada en fuentes documentales de diversa procedencia; por un lado, se localizan referencias en valoraciones regionales del estado de los stocks, fundamentalmente atuneros; también, en segundo lugar, en evaluaciones ex post de ACPS comunitarios en los que se da noticia de la actividad pesquera existente en Estados colindantes; y, sobre todo, en las evaluaciones ex ante que resultan preceptivas

7 En virtud del Reglamento (CE) 1006/2008 del Consejo, de 29 de septiembre de 2008, relativo a la autorización de las actividades pesqueras de los buques pesqueros comunitarios fuera de las aguas comunitarias y al acceso de los buques de terceros países a las aguas comunitarias (DO L 286, 29-10-2008) que acaba de ser derogado.

8 El Consejo Consultivo de la Flota de Larga Distancia (Long Distance Advisory Council, LDAC) se ha referido a la necesidad de acabar con esta dualidad de regímenes en sus recomendaciones sobre la dimensión externa de la PPC al indicar que «the levels of transparency required for the activities of the European fleets that operate under agreements must be applied to the activities of the European fleets that operate outside of the fisheries partnership agreements (joint ventures, charters, private agreements)», (LDAC, 2015). 
para sopesar la pertinencia o bien de suscribir un ACPS con países ribereños con los que la UE no ha mantenido previamente relaciones convencionales en el ámbito pesquero o bien, si ya existe tratado en vigor, de firmar un nuevo protocolo. A través de estos tres subconjuntos de documentación técnica se puede rastrear la existencia de acuerdos privados suscritos por armadores europeos, en particular franceses y españoles, en Guinea, Madagascar y Nauru', Liberia, Sierra Leona, Tokelau y Tuvalu ${ }^{10}$ y, por último, Kenia, Mauricio, Senegal y Tanzania ${ }^{11}$.

En una penumbra semejante a la que rodea a las licencias privadas de pesca en aguas extracomunitarias se encuentra la información disponible sobre los denominados acuerdos de flete (chartering agreements). En esta modalidad de acceso, los buques de bandera de un Estado miembro de la UE son contratados por las empresas locales del Estado ribereño - que cuentan con derechos de pesca pero carecen de capacidad técnica para explotarloscon el objetivo de hacerlos partícipes de las actividades extractivas realizadas en la ZEE del país. Los acuerdos de chárter pueden adoptar un formato de mínimos, en el supuesto de que solo sea objeto de arriendo por un tiempo determinado el buque a través de un "contrato a casco desnudo" (bareboat charter), o de máximos, en el caso de que el alquiler del buque comunitario, que mantiene en todo momento su pabellón de origen, se realice con tripulación.

\section{LA INCLUSIÓN DE CLÁUSULAS DE EXCLUSIVIDAD EN LOS ACPS}

Las cláusulas de exclusividad son dispositivos normativos orientados hacia el control del esfuerzo pesquero realizado por la flota comunitaria en aguas de terceros Estados puesto que, una vez que entra en vigor el acuerdo entre la Unión y un país asociado, los buques de la UE solo pueden operar en las aguas del Estado contraparte si cuentan con una autorización de pesca que haya sido emitida en virtud de un protocolo de aplicación del tratado. La finalidad que persigue la incorporación de este tipo de cláusulas en los acuerdos es, por consiguiente, «reforzar el marco de gobernanza para las actividades de pesca de la flota de aguas distantes de la UE» (Tribunal de Cuentas Europeo, 2015: 11).

9 Respectivamente en COFREPECHE et al. (2013b); POSEIDON et al. (2014b); POSEIDON et al. (2013).

10 COFREPECHE et al. (2012); POSEIDON et al. (2012); Oceanic Development y Megapesca (2014b).

11 POSEIDON et al. (2014a); Oceanic Development y Megapesca (2011); COFREPECHE et al. (2015); COFREPECHE et al. (2013a); NFDS et al. (2014). 
Las cláusulas de exclusividad fueron empleadas con menos asiduidad en los tratados de carácter básicamente comercial anteriores a 2004, pero, tras la adopción de los acuerdos de asociación pesquera, este tipo de mecanismo se generalizó. En consecuencia, el Reglamento de base de la PPC solo ha venido a consolidar jurídicamente un mecanismo preexistente mediante el que se busca asegurar un mejor control de la presión extractiva a la que se encuentran sometidos los recursos de la ZEE del Estado ribereño ${ }^{12}$. El mandato relativo a la incorporación de la cláusula de exclusividad en los nuevos ACPS resulta coherente tanto con el énfasis sobre la dimensión externa (por primera vez contemplada en la normativa básica de esta política común) que se realiza durante la última revisión de la $\mathrm{PPC}^{13}$ como con la filosofía que inspira la remozada acción exterior pesquera, puesto que la UE busca no solo mejorar la gestión de sus propios espacios marinos, sino también "promover los objetivos de la PPC a nivel internacional, velando por que las actividades pesqueras de la Unión en aguas ajenas a la Unión se basen en los mismos principios y normas que la legislación aplicable de la Unión» ${ }^{14}$.

12 El Reglamento (UE) 1380/2013 establece que «los buques de pesca de la Unión solo podrán faenar en las aguas del tercer país con el que esté vigente un acuerdo de colaboración de pesca sostenible si están en posesión de una autorización de pesca expedida con arreglo a un procedimiento convenido en el acuerdo» (art. 31.5) e indica, además, que los ACPS deben incluir, en la medida de lo posible, una cláusula de exclusividad (art. 31.6.b).

13 La inserción de la parte VI (Política exterior) en el Reglamento de base 1380/2013, arts. 28-33, constituye una de las principales novedades de la última reforma de la PPC. Para una revisión de conjunto sobre el alcance de la dimensión externa de esta política, véase Penas Lado (2016: 144-169).

14 Considerando 50 del Reglamento (UE) 1380/2013, loc.cit. Para un análisis más omnicomprensivo (y, por tanto, que trasciende la dimensión pesquera) de la estrategia general de la UE relativa a la gestión oceánica, véase el reciente documento Comisión Europea y Alta Representante de la Unión para Asuntos Exteriores y Política de Seguridad, Comunicación conjunta al Parlamento Europeo, al Consejo, al Comité Económico y Social Europeo y al Comité de las Regiones sobre la Gobernanza internacional de los océanos: una agenda para el futuro de nuestros océanos, Bruselas, 10-11-2016 JOIN(2016) 49 final. En el mismo sentido, es preciso indicar (como ha sido observado durante el proceso de evaluación anónima de este trabajo) que la UE está impulsando la adopción de un acuerdo de aplicación del CNUDM en materia de conservación y uso sostenible de la diversidad marina en aguas internacionales, uno de cuyos aspectos clave será el desarrollo de capacidades y la transferencia de tecnología hacia los países en desarrollo, lo que repercutirá positivamente sobre la correcta gestión de las ZEE por parte de estos. 
Para comprender los motivos que explican la inserción de cláusulas de exclusividad en los ACPS es preciso tener en cuenta que, aunque la gestión de la pesca en aguas comunitarias constituye el modelo referencial para la celebración por parte de la UE de acuerdos internacionales con terceros Estados, no es posible extrapolar una parte de los instrumentos de regulación empleados a nivel interno a aguas extracomunitarias, como ocurre, entre otros, con la fijación de totales admisibles de captura ${ }^{15}$. La falta de capacidad administrativa y técnica de la mayoría de los países en desarrollo con los que se suscriben los ACPS dificulta la implementación de los principios de la buena gobernanza en la dimensión exterior de la PPC. La propia determinación del excedente existente en las ZEE de los Estados contraparte —que resulta fundamental para la correlativa fijación de las posibilidades de pesca disponibles para la flota comunitaria- plantea problemas estructurales de difícil resolución. El Tribunal de Cuentas Europeo ha insistido en denunciar que las evaluaciones ex post ponen de manifiesto que los países socios carecen de la capacidad de determinar el excedente, sobre todo en el caso de acuerdos mixtos o de recursos de pesca muy dispersos, como las especies pelágicas o las especies altamente migratorias (Tribunal de Cuentas Europeo, 2015: 38). Al problema de la falta de capacidad técnica del ribereño se añade, en la mayoría de las ocasiones, la inexistencia de información sobre el esfuerzo pesquero realizado por la propia flota nacional del Estado o por otros buques extranjeros a los que el país socio también haya concedido acceso ${ }^{16}$.

15 Desde esa perspectiva más modesta, el Reglamento de base se limita a establecer la pauta de que «los acuerdos de colaboración de pesca sostenible con terceros países deben garantizar que las actividades pesqueras de la Unión en aguas de terceros países se basen en los mejores dictámenes científicos disponibles y en el intercambio de información pertinente, garantizando una explotación sostenible de los recursos biológicos marinos, la transparencia por lo que respecta a la determinación del excedente, y la consiguiente gestión de los recursos de manera coherente con los objetivos de la PPC», considerando 51, loc.cit.

16 La incertidumbre que genera la falta de información sobre las capturas realizadas por otras flotas extranjeras en la ZEE del ribereño está intentando ser reducida por la UE, en los últimos años, a través de la inclusión de disposiciones en los nuevos acuerdos que obliguen al país contraparte a compartir dicha información. De esta forma, el art. 1.6 del vigente Protocolo con Mauritania señala que «Mauritania se compromete a hacer público todo acuerdo público o privado que autorice el acceso de buques extranjeros a su ZEE, en particular: el período o períodos cubiertos por el Acuerdo; el número de buques y los tipos de artes autorizados; las especies o poblaciones autorizadas para la pesca, incluido todo límite de captura aplicable; las medidas de declaración, seguimiento, control y vigilancia requeridas; una copia del acuerdo escrito", 
Atendiendo a lo indicado, por tanto, resulta evidente que la mayoría de los Estados contrapartes poseen una capacidad limitada para gestionar la gobernanza pesquera de sus ZEE. Desde esa perspectiva, se comprende mejor que la cláusula de exclusividad haya sido concebida como un dispositivo de autolimitación de la flota comunitaria que está llamado, en principio, a generar efectos claramente positivos, puesto que permite determinar con precisión el esfuerzo pesquero real que realizan los buques con pabellón de los Estados miembros de la UE en un caladero dado.

La inclusión gradual de la cláusula de exclusividad que se ha realizado en los acuerdos pesqueros nos permite delinear algunas de las características de estas disposiciones en la práctica comunitaria. Un primer rasgo a reseñar es que el formato de redacción de la cláusula tiende a diferir en el ámbito de los acuerdos atuneros, donde ha sido delimitada de manera estricta para que solo se permita faenar a buques comunitarios al amparo del convenio, y en el plano de los acuerdos mixtos en los que la prohibición aparece con frecuencia matizada, con la intención de no clausurar completamente la posibilidad de que otros buques con bandera de Estados miembros que no estén cubiertos por el convenio puedan llegar a explotar, eventualmente, aquellas especies que no hayan sido objeto de regulación en el tratado ${ }^{17}$. Una segunda nota a destacar, también de carácter formal, es que, a partir de la entrada en vigor del Reglamento de base de 2013, da la sensación de que el legislador comunitario no se conforma con explicitar la obligación de incluir cláusulas de exclusividad en los nuevos acuerdos pesqueros que suscriba la UE, sino que busca, además, depurar la formulación de estas disposiciones. Así se pone de manifiesto en el convenio con Senegal, que ha sido el primer ACPS adoptado

DO L 315, 1-12-2015. El Parlamento Europeo ha indicado respecto a esta cuestión que «anima encarecidamente a la Comisión a que vele por la inclusión de disposiciones sobre transparencia similares en otros protocolos futuros, con lo que se mejoraría considerablemente la transparencia en relación con el esfuerzo de pesca total y las condiciones de acceso", Parlamento Europeo, Informe sobre un régimen común con vistas a aplicar la dimensión exterior de la PPC, incluidos los acuerdos de pesca, A80052/2016, 10-3-2016, párr. 49.

17 Encontramos esta situación, por ejemplo, en los tratados celebrados con Guinea-Bisáu (DO L342, 27-12-2007, art. 6.2) o Mauritania (DO L343, 8-12-2006, art. 6.2). $\mathrm{El}$ art. 6 del acuerdo con Marruecos nos permite ejemplificar esta práctica cuando señala que «cuando se trate de categorías de pesca que no estén contempladas en el Protocolo vigente, las autoridades marroquíes podrán conceder licencias a buques comunitarios. No obstante, y de conformidad con el objetivo de colaboración establecido por el presente Acuerdo, la concesión de dichas licencias estará supeditada a la recepción de un dictamen favorable de la Comisión Europea», DO L 141, 29-5-2006. 
tras la reforma de la PPC, en el que la cláusula diseñada prohíbe, de manera expresa y no tácita, la existencia de autorizaciones pesqueras que deriven de acuerdos privados ${ }^{18}$.

El tercer y último elemento — de carácter más sustantivo- que se puede extraer de la práctica reciente relativa a este tipo de disposiciones se refiere a la interpretación rigorista de la cláusula de exclusividad que ha hecho el TJUE en la relevante sentencia dictada en el asunto Ablström y otros, mediante la que se resolvía la controversia planteada por dos navíos suecos fletados (con un contrato "a casco desnudo») por una empresa marroquí para explotar, en la costa del Sáhara Occidental, cuotas nacionales al amparo de una licencia expedida por las autoridades marroquíes ${ }^{19}$. En este contexto mercantil de relaciones contractuales establecidas entre particulares, en opinión de los armadores suecos —que se encontraban incursos en un procedimiento penal por pesca ilegal ante su jurisdicción nacional, desde la que fue suscitada la cuestión prejudicial resuelta por el TJUE— no tendría por qué producirse intervención alguna ni por parte de las autoridades del Estado de pabellón ni tampoco de las comunitarias, careciendo de relevancia las disposiciones relativas a la cláusula de exclusividad recogidas en el acuerdo pesquero con Marruecos ${ }^{20}$. El Tribunal, sin embargo, ha rechazado de plano esta argumentación al entender que la vigencia de esta clase de disposiciones limita toda actividad pesquera por parte de buques con pabellón de un Estado miembro de la UE en las aguas del Estado contraparte, no solo bajo la forma de acuerdos privados suscritos directamente por los armadores comunitarios con las autoridades del ribereño, sino también en el caso más extremo de que los

18 En el art. 4.2 del acuerdo se aclara, a fin de despejar toda ambigüedad que pudiera albergar la formulación tradicional de la cláusula, que «las autoridades senegalesas únicamente expedirán autorizaciones de pesca en virtud del presente Acuerdo a los buques pesqueros de la Unión y tendrán prohibido expedir autorizaciones a dichos buques fuera de este marco, en particular en forma de licencias privadas», DO L 304, 23-10-2014. La referencia explícita a la prohibición de las licencias privadas no se incluía en los acuerdos firmados con anterioridad a 2013.

Sentencia del Tribunal de Justicia, Ahlström y otros, C-565/13, EU:C:2014:2273. Para un comentario sobre la sentencia, véase Oanta (2016).

20 Los armadores alegaron, siguiendo el razonamiento, que los buques de pesca Aldo y Nordic IV fueron arrendados a la sociedad marroquí Atlas Pelagic, que disponía de sus propios derechos de pesca en las aguas territoriales marroquíes, utilizándolos esta de modo autónomo en el marco de sus actividades, por lo que ni el derecho de la Unión ni el derecho pesquero sueco resultaban aplicables a la actividad de arrendamiento ejercida por las empresas (Fiskeri Ganthi y Fiskeri Nordic) de las que eran propietarios, ibid., párr. 20. 
navíos con bandera de un Estado miembro hayan sido «charteados» — como en este asunto- por empresas del país ribereño con el objetivo de explotar cuotas propias de carácter nacional ${ }^{21}$.

\section{EL PERNICIOSO EFECTO COLATERAL DE LAS CLÁUSULAS DE EXCLUSIVIDAD SOBRE LOS ACUERDOS DURMIENTES}

Además de los doce ACPS que la UE mantiene activos mediante protocolos de aplicación ${ }^{22}$, llama poderosamente la atención en la práctica convencional comunitaria la pervivencia de ocho acuerdos durmientes en los que los instrumentos de ejecución, por motivos diversos, no han sido renovados con cinco países contrapartes africanos y tres Estados insulares del Pacífico. Cuando se plantea una situación de esta naturaleza, es esencial subrayar que la cláusula de exclusividad continúa desplegando efectos jurídicos con plenitud y que, en consecuencia, ningún buque que porte pabellón de un Estado miembro de la UE puede realizar actividad pesquera alguna (ni siquiera mediante otro mecanismo alternativo como las licencias privadas o los contratos de chárter) en las aguas jurisdiccionales del Estado ribereño; en consecuencia, esta restricción se proyecta todavía, a noviembre de 2017, sobre las ZEE de las Comoras, Gabón, Gambia, Guinea Ecuatorial, Kiribati, Micronesia, Mozambique y las islas Salomón.

21 El TJUE recuerda, en particular, que si bien el art. 6, apdo. 2, del Acuerdo de pesca establece que las autoridades marroquíes podrán conceder licencias a buques comunitarios para las categorías de pesca que no estén contempladas en el protocolo, la concesión de dichas licencias «estará supeditada a la recepción de un dictamen favorable de la Comisión Europea. De lo anterior se deriva que, para que un buque comunitario pueda desarrollar actividades de pesca en las zonas de pesca marroquíes, siempre es necesaria una intervención de las autoridades competentes de la Unión y, en consecuencia, dicho buque no podrá ejercer dichas actividades en las zonas mencionadas sobre la base de una licencia expedida por las autoridades marroquies competentes sin tal intervención" (la cursiva es mía, párr. 28-29). En consecuencia, no puede admitirse que los buques comunitarios accedan a las zonas de pesca marroquíes para realizar en ellas actividades de pesca mediante la celebración con este fin de un contrato de fletamento con una sociedad marroquí que posee una licencia expedida por las autoridades marroquíes en favor de titulares marroquíes de cuotas de pesca, ni mediante la utilización de cualquier otro instrumento jurídico que les permita acceder a dichas zonas de pesca para ejercer en ellas tales actividades fuera del marco del Acuerdo de pesca y, por tanto, sin la intervención de las autoridades competentes de la Unión, ibid., párr. 33).

22 Véase supra apdo. I.1. 
Ante esta circunstancia, resulta posible deslindar dos casuísticas diferentes. En primer término, la de aquellos acuerdos cuyos protocolos han expirado en fechas relativamente recientes, como puede ser el caso de Gabón y las Comoras en 2016 o los de Kiribati y Mozambique en 2015. En algunos de estos casos existen negociaciones en marcha para la renovación de protocolos, que pueden resultar más o menos exitosas en función de los intereses concretos en presencia, como ha ocurrido respecto a Mozambique, donde han sido abandonadas por falta de un acuerdo económico entre las partes. En otros supuestos, los motivos que obstaculizan la adopción de un nuevo protocolo tienen un carácter netamente político, como ocurre con Gabón ${ }^{23}$, o, incluso, pueden ser de tipo técnico pesquero, como sucede en el caso de las Comoras ${ }^{24}$.

23 Parlamento Europeo, Resolución sobre la represión de la oposición en Gabón, 14-92017, P8_TA(2017)0349.

24 El tratamiento dado a la relación bilateral pesquera con las Comoras ha sido excepcional y merece una valoración positiva al ejemplificar una línea de coherencia que la Comisión Europea debiera extender a la gestión de toda la dimensión exterior de la PPC. En este caso, la Comisión acaba de solicitar al Consejo la denuncia del acuerdo pesquero con el país al considerarlo como «no cooperante» en la lucha contra la pesca ilegal, no declarada y no reglamentada (INDNR), véase Comisión Europea, Propuesta de Decisión del Consejo por la que se denuncia el Acuerdo de colaboración en el sector pesquero entre la Comunidad Europea y la Unión de las Comoras, adoptado por el Reglamento (CE) 1563/2006 del Consejo, de 5 de octubre de 2006, Bruselas, 29-9-2017 COM(2017) 556 final. Con arreglo al art. 31 del Reglamento (CE) 1005/2008 del Consejo ("el Reglamento INDNR»), la Comisión Europea puede identificar a los terceros países que en su opinión no cooperan en la lucha contra la pesca INDNR. Se puede considerar como no cooperante al país que no cumpla la obligación de adoptar medidas para prevenir, desalentar y eliminar la pesca INDNR que, en virtud del derecho internacional, le incumbe en su calidad de Estado de abanderamiento, Estado rector del puerto, Estado ribereño o Estado de comercialización. De conformidad con el art. 32 del Reglamento INDNR, el 1 de octubre de 2015, mediante una Decisión de la Comisión, se notificó a las Comoras la posibilidad de que fuera considerado tercer país no cooperante en la lucha contra la pesca ilegal, no declarada y no reglamentada (DO C 324 de 2-10-2015, p. 6). A raíz de dicha decisión, la Comisión abrió un diálogo con las Comoras, que se llevó a cabo de conformidad con los requisitos establecidos en el Reglamento INDNR y se basó en un plan de acción propuesto por la Comisión para subsanar las deficiencias. Las Comoras no adoptaron, dentro de un plazo razonable, las medidas correctoras necesarias para resolver los problemas detectados, por lo que, mediante la Decisión de Ejecución (UE) 2017/889 de la Comisión, de 23 de mayo de 2017, dicho país fue identificado, de conformidad con el art. 31 del Reglamento INDNR, como tercer país no cooperante (DO L 135 de 24-5-2017, p. 35). Tras dicha identificación, las Comoras tampoco adoptaron las 
De cualquier manera, los problemas que se derivan de la existencia de un breve período de discontinuidad entre la vigencia de dos protocolos no suelen ser graves y han sido subsanados de acuerdo con la legalidad comunitaria aplicable, puesto que el art. 9 del recientemente derogado Reglamento sobre autorizaciones pesqueras contenía la previsión de que la Comisión podía —en los casos en que hubiese expirado un protocolo y ya hubiese sido rubricado uno nuevo, sin que el Consejo lo hubiese firmado todavía, o bien en el supuesto de que hubiese sido establecida la aplicación provisional del mismo- transmitir las solicitudes de autorización de pesca al tercer país afectado, durante un período de seis meses a partir de la fecha de expiración del protocolo anterior ${ }^{25}$. Esta solución, no exenta de dificultades burocráticas (como se demostró en el caso de la terminación del Protocolo 2008-2012 con Mauritania) ha constituido una vía habitual para subsanar las cesuras temporales que aparecen entre protocolos ${ }^{26}$.

Sin embargo, quisiéramos centrar la atención sobre la problemática más grave que plantean, en segundo término, aquellos acuerdos pesqueros que han permanecido carentes de protocolo de aplicación durante un período superior a los tres años, que es a los que debemos calificar como realmente durmientes. En este supuesto nos encontramos con los casos de islas Salomón

medidas correctoras necesarias por lo que, mediante la Decisión de Ejecución (UE) 2017/1332 del Consejo (DO L 185 de 18-7-2017, p. 37), se añadió al Estado a la lista de terceros países no cooperantes establecida mediante la Decisión de Ejecución 2014/170/UE (DO L 91 de 27-3-2014, p. 43) para que finalmente, como desenlace del proceso, se haya iniciado el proceso de denuncia del convenio. Esta rápida respuesta de la Comisión (que se compadece mal con la pasividad mostrada, como veremos, con los acuerdos durmientes) resulta acorde, sin embargo, con la posición de liderazgo internacional en la lucha contra la pesca INDNR que ha asumido la UE a lo largo de la última década.

25 Reglamento (CE) 1006/2008 del Consejo, de 29 de septiembre de 2008, relativo a la autorización de las actividades pesqueras de los buques pesqueros comunitarios fuera de las aguas comunitarias y al acceso de los buques de terceros países a las aguas comunitarias, DO L 286, 29-10-2008.

26 En el caso mencionado, las autoridades mauritanas consideraron que las licencias concedidas para el período de intermedio entre protocolos (entre agosto y diciembre de 2011) tenían que ser tomadas como una medida de aplicación provisional del protocolo firmado para el período 2012-2014 y, en consecuencia, concluyeron que las licencias pagadas por los armadores hasta la fecha de expiración real del acuerdo (1512-2014) debían finalizar anticipadamente, a fin de computar la actividad realizada durante los meses de transición entre protocolos, véase Tribunal de Cuentas Europeo (2015: 15). 
(desde el 9-10-2012), Micronesia (desde el 25-2-2010) y, de manera todavía más significativa, Guinea Ecuatorial (desde el 30-6-2001) y Gambia (desde el 1-71996). Cuando se produce esta anómala circunstancia, los efectos que se generan para la flota de aguas exteriores de la UE resultan particularmente perniciosos, puesto que los buques de bandera comunitaria ven bloqueado - por efecto de la cláusula de exclusividad y, en ocasiones extremas, durante períodos que alcanzan hasta los veinte años- su acceso a las pesquerías del país ribereño a través de cualquier otra solución jurídica, como podrían ser las licencias privadas.

Ante este panorama, la vigencia de las cláusulas de exclusividad en los acuerdos durmientes aboca a tres posibles escenarios. El primero consiste, sin más, en conseguir que la flota comunitaria no pesque en dichas aguas, lo que resulta pertinente en términos jurídicos para la UE pero deviene completamente antieconómico para armadores y pescadores cuando la situación de bloqueo se enquista y se mantiene estancada durante décadas. La segunda opción pasa por reabanderar los buques bajo un pabellón extracomunitario que no se encuentre vinculado por la cláusula de exclusividad, bien el del Estado ribereño en cuestión o bien una bandera de conveniencia; esta solución, que se ha suscitado con cierta frecuencia en nuestra práctica pesquera ${ }^{27}$, resulta también indeseable no solo para la UE sino también para el armador, puesto que implica para este tanto una merma de seguridad jurídica como la necesidad de afrontar las dificultades burocráticas aparejadas al eventual retorno del navío al registro comunitario.

La tercera alternativa que ha sido articulada en la práctica, como respuesta a la paradoja que plantean los acuerdos durmientes, consiste en saltarse la normativa e ignorar la cláusula de exclusividad. La información disponible sobre la vulneración del mandato inherente a estas disposiciones resulta escasa, pero, a través de diversas fuentes indirectas, podemos rastrear de manera indiciaria el alcance de la infracción. En el caso de Guinea Ecuatorial, por ejemplo, donde el acuerdo lleva latente diecisiete años, se ha puesto de manifiesto, en la evaluación prospectiva sobre la eventual celebración de un nuevo

27 La Comisión ya alertaba tempranamente de dicha posibilidad al advertir, en su toma de posición sobre la dimensión externa de la PPC previa a la reforma del Reglamento de base, de que "para prevenir la elusión de la cláusula de exclusividad mediante el reabanderamiento, los futuros acuerdos pesqueros deben prever que un buque de la UE que cambie de pabellón para eludir sus obligaciones o conseguir más posibilidades de pesca no esté autorizado a pescar más en la ZEE del país asociado», Comisión Europea, Comunicación de la Comisión al Parlamento Europeo, al Consejo, al Comité Económico y Social Europeo y al Comité de las Regiones sobre la dimensión exterior de la Política Pesquera Común, COM (2011) 424 final, 13-7-2011, p. 13. 
protocolo de aplicación, que un contingente de atuneros cerqueros obtuvo licencias para faenar en dichas aguas, a través del establecimiento de acuerdos privados con el ministerio ecuatoguineano de pesca, por lo que la Comisión Europea se vio obligada, en 2014, a recordar a los Estados miembros la necesidad de respetar la vigencia de la cláusula de exclusividad del convenio establecido en $1984^{28}$.

En el mismo sentido, cabe señalar que el intento más ambicioso de arrojar luz sobre la vulneración de la cláusula de exclusividad ha correspondido hasta la fecha a la ONG Oceana, que, empleando datos procedentes de Global Fishing Watch ${ }^{29}$, afirma haber localizado a diecinueve buques de bandera comunitaria (con pabellones de Grecia, Italia, Portugal y España) que faenaron más de 31000 horas en las ZEE de Guinea Ecuatorial y de Gambia entre abril de 2012 y agosto de 2015 (OCEANA, 2017). Si bien estos datos tienen un carácter estimativo y pueden ser discutidos en cuanto su precisión ${ }^{30}$, lo

28 De acuerdo con el informe, 《las capturas de los barcos de la UE que estuvieron activos en las aguas ecuatoguineanas serían de 3550 toneladas anuales de media para el periodo 2008-2013, según los datos de captura enviados a ICCAT», COFREPECHE et al. (2016: 7).

29 Global Fishing Watch es un proyecto no gubernamental que 《uses data and open technologies to help save the oceans by showing, for free, exactly where all of the trackable commercial fishing activity has happened since 2012 and is happening today in near-real time. The Global Fishing Watch Map represents billions of publicly-broadcast Automatic Identification Signals (AIS) from ships at sea. Artificial intelligence tools developed by our data scientists, process these signals to distinguish individual vessel tracks and identify when the vessels are fishing based on their movements", http://globalfishingwatch.org/the-project (consultado el 29-9-2017). Sobre la base técnica de la generación de datos útiles para el análisis de la gobernanza pesquera, véase Jablonicky et al. (2016), así como Robards et al. (2016).

30 La propia Oceana abre su análisis planteando una advertencia sobre el carácter oficioso de los datos que emplea, al admitir que «any and all references to «fishing» should be understood in the context of Global Fishing Watch's fishing detection algorithm, which is a best effort to determine «apparent fishing effort» based on vessel speed and direction data from the Automatic Identification System (AIS) collected via satellites and terrestrial receivers. As AIS data varies in completeness, accuracy and quality, it is possible that some fishing effort is not identified and conversely, that some fishing effort identified is not fishing. For these reasons, Global Fishing Watch qualifies all designations of vessel fishing effort, including synonyms of the term «fishing effort,» such as "fishing» or "fishing activity," as «apparent,» rather than certain. Any/all Global Fishing Watch information about "apparent fishing effort» should be considered an estimate and must be relied upon solely at your own risk», OCEANA (2017: 1). 
cierto es que constituyen un indicador inequívoco de la existencia de actividad pesquera comunitaria en las ZEE de países con acuerdos durmientes en vigor.

Los rastros de esta actividad pueden intuirse también en las evaluaciones ex ante y ex post del protocolo con Mozambique en vigor entre 20122015, en el que se recogió la denuncia de que buques camaroneros de bandera portuguesa habían operado bajo acuerdos de chárter y al amparo de licencias de compañías mozambiqueñas, en vulneración también de la estricta cláusula de exclusividad del acuerdo (Oceanic y Megapesca, 2014a), y en diversas informaciones periodísticas que dan cuenta de la actividad de buques de pabellón comunitario en zonas de pesca en las que, en principio, tienen prohibido faenar ${ }^{31}$.

\section{UNA VENTANA DE OPORTUNIDAD PARA RESOLVER LA PROBLEMÁTICA VINCULADA A LOS ACUERDOS DURMIENTES: EL PROCEDIMIENTO LEGISLATIVO DE ADOPCIÓN DEL REGLAMENTO (UE) 2017/2403 SOBRE LA GESTIÓN SOSTENIBLE DE LAS FLOTAS PESQUERAS EXTERIORES}

Debido a la escasa visibilidad del problema generado por los efectos de las cláusulas de exclusividad en los acuerdos durmientes, la Comisión Europea se ha mostrado al respecto radicalmente inoperativa, limitándose a dejar transcurrir el tiempo sin poner remedio a la situación. Durante el último año, sin embargo, la cuestión ha adquirido una mayor relevancia en la agenda política comunitaria, al haberse abierto una ventana de oportunidad para su resolución. El contexto que ha propiciado esta coyuntura ha sido la iniciativa de la Comisión de impulsar la adopción de un nuevo Reglamento sobre la

31 En ese sentido, podemos constatar, a título ilustrativo, cómo en una noticia relativa a la búsqueda de caladeros alternativos por parte de la flota cefalopodera gallega, tras su salida forzosa de Mauritania, se informa de que «en Angola, a través de licencias privadas, faenan tres embarcaciones, mientras que en Gambia trabajan actualmente cuatro" (La Voz de Galicia, 2-2-2015), a pesar de que este último Estado ribereño tiene suscrito un acuerdo durmiente con la UE. En otro rotativo, que realiza un seguimiento de la actualidad relativa a la labor cotidiana de la flota de aguas exteriores, se informa, también, en una pieza que lleva por título «Gambia, un caladero de solo 34 millas, única alternativa al «atropello» de Guinea Conakry», que "cinco barcos cefalopoderos gallegos, expulsados con el resto de esta flota de arrastreros de Mauritania el pasado año, fían en estos momentos su suerte al «diminuto» caladero de Gambia», (El Faro de Vigo, 26-3-2014). 
gestión sostenible de las flotas pesqueras exteriores ${ }^{32}$ que, tras su adopción y entrada en vigor, ha supuesto la derogación del Reglamento (CE) 1006/2008 sobre autorizaciones pesqueras, con el objetivo principal de dar cumplimiento al mandato del Reglamento de base de la PPC que establece que la Unión debe llevar a cabo la supervisión de su flota allí donde se encuentre y, sobre todo, con independencia del marco normativo en el que se inscriban sus actividades ${ }^{33}$.

Hasta comienzos de 2018, la Comisión Europea solo ha tenido poderes para supervisar la actividad de los buques de pabellón de un Estado miembro que faenaban en aguas extracomunitarias al amparo de acuerdos pesqueros de carácter público, pero no podía controlar la pesca realizada a través de licencias privadas o acuerdos de flete puesto que, de acuerdo con el Reglamento (CE) 1006/2008, en estos casos los Estados miembros de pabellón solo debían comunicar a la Comisión Europea el nombre de los navíos implicados, sin aportar otros datos relevantes como puede ser la especie objetivo, la zona de pesca, el período o las artes de pesca utilizadas ${ }^{34}$.

32 Comisión Europea, Propuesta de Reglamento del Parlamento Europeo y del Consejo sobre la gestión sostenible de las flotas pesqueras exteriores y por el que se deroga el Reglamento (CE) 1006/2008 del Consejo, COM(2015) 636 final, 10-12-2015

El Reglamento (UE) 2017/2403 ha entrado en vigor el 19-1-2018, durante el proceso de revisión de este artículo.

34 Para ser ecuánimes, es preciso señalar que, a pesar de que el marco normativo comunitario ha ejercido un control laxo sobre este tipo de acuerdos, la Administración pesquera española fue precursora a la hora de implementar mecanismos de fiscalización sobre las licencias privadas, que han terminado funcionado como antecedentes del nuevo panorama normativo para el control de la flota exterior que la UE ha definido mediante el Reglamento (UE) 2017/2403. La Administración española ha defendido que a los buques comunitarios que desarrollan su actividad bajo licencias privadas en países terceros se les deben exigir los mismos requisitos que a los buques que operan en el marco de acuerdos pesqueros concluidos por la UE. En este sentido, aparte de las preceptivas licencias emitidas por el tercer país, todos los navíos españoles están obligados a obtener un permiso temporal expedido por la Secretaría General de Pesca, que incluye la exigencia de aplicación de toda la normativa pertinente tanto de la UE como de las Organizaciones Regionales Pesqueras, así como la normativa nacional relevante. A fin de garantizar que los buques que obtienen licencias privadas en terceros países operan con seguridad jurídica y de evitar posibles problemas de falsedad de licencias o de intervención de agentes no autorizados, la Administración española vienen aplicando, ya desde finales de 2012, un sistema de verificación de licencias a través de la red de embajadas españolas y delegaciones de la UE en los terceros países. 
El Reglamento (UE) 2017/2403 ha sido adoptado para atacar este déficit de control mediante la introducción de una serie de novedades regulatorias como son, entre otras, que el navío comunitario deberá tener una autorización de pesca emitida por su Estado de pabellón (además de la licencia privada que le expida el tercer Estado) o también que el armador deberá identificar, a través del número OMI, tanto al pesquero beneficiario de la licencia como a cualquier otro buque de apoyo que faene al amparo de un acuerdo privado. El núcleo de la reforma realizada ha consistido, por tanto, en garantizar que - con independencia de cuál sea el marco operativo de un buque de pabellón de un Estado miembro fuera de aguas comunitarias (en alta mar bajo gestión, o no, de una OROP, bajo el régimen de un ACPS, al amparo de una licencia privada o mediante un contrato de flete) - existe una capacidad efectiva de control y seguimiento tanto por parte del Estado de abanderamiento como por la Comisión Europea ${ }^{35}$. Este objetivo legislativo está en plena consonancia con las «Directrices voluntarias de la FAO para la actuación del Estado del pabellón», destinadas a prevenir, desalentar y eliminar la pesca INDNR ${ }^{36}$, así como con el principio de «debida diligencia» en materia de pesca que el Tribunal Internacional del Derecho del Mar ha puntualizado que ha de observar la Unión Europea respecto al control de las obligaciones que incumben a los países miembros en tanto que Estados de pabellón ${ }^{37}$.

La primera lectura del Parlamento Europeo (PE) sobre la propuesta del nuevo reglamento se saldó con la adopción de una resolución legislativa apoyada por 586 votos, con 56 en contra y 6 abstenciones, que avaló la iniciativa de la Comisión, si bien introdujo modificaciones sobre varios

35 Como ha indicado la Comisión «il apparait nécessaire à la fois d'accroître la cohérence entre les différents instruments de suivi, de contrôle et de surveillance des activités de pêche exercées par des navires de l'Union européenne, de remédier aux incertitudes juridiques et aux lacunes du règlement actuel, et enfin, le cas échéant, de renforcer le cadre réglementaire en assurant le respect de règles comparables par l'ensemble du secteur de la pêche de l'Union européenne opérant dans les eaux extérieures à l'Union, notamment en améliorant le suivi des autorisations de pêche privées et en prévenant les changements abusifs de pavillon", Comisión Europea, Analyse d'impact..., loc. cit., pp. 9-10.

36 Las directrices fueron ratificadas por el COFI en su trigésimo primera sesión, celebrada en Roma del 9 al 13 de junio del 2014, véase FAO (2015). Directrices Voluntarias para la Actuación del Estado del Pabellón, Roma, 53 pp.

37 International Tribunal for the Law of the Sea (ITLOS), Request for an advisory opinion submitted by the Sub-Regional Fisheries Commission, Advisory Opinion, 2 April 2015. Al respecto, veánse García Andrade (2016); Jorge Urbina (2017). 
aspectos de la misma ${ }^{38}$. El PE planteó, entre otras cuestiones, que los controles establecidos sobre el proceso de reabanderamiento deben ser aplicables a los buques que han abandonado el registro comunitario y desean regresar al mismo solo durante los dos años que preceden a la solicitud de reincorporación, al estimar excesivo el plazo de cinco años que figuraba en el art. 6 de la propuesta original de la Comisión; sobre los ACPS, los parlamentarios solicitaron también que una parte proporcional del apoyo sectorial prestado al Estado ribereño contraparte sea reservado para financiar la integración de estos países en las OROP regionales que les afecten; por otra parte, la resolución parlamentaria estableció que si la Comisión exigía una evaluación científica que demostrase la sostenibilidad de las actividades propuestas en el marco de acuerdos privados, por coherencia lógica debería exigir un requisito análogo para los buques que pescan en alta mar pero fuera del alcance de una OROP.

Después de haber desarrollado tres trílogos negociadores, la Comisión, el Parlamento y el Consejo alcanzaron un acuerdo sobre la redacción final del Reglamento (UE) 2017/2403, que encontró reflejo formal tanto en la primera lectura de la propuesta realizada por el Consejo como en la segunda (y definitiva) lectura parlamentaria ${ }^{39}$. Las principales conclusiones alcanzadas en la recta final de la negociación se refieren a que, a la postre, el control

38 Parlamento Europeo, Comisión de Pesca, Informe sobre la propuesta de Reglamento del Parlamento Europeo y del Consejo sobre la gestión sostenible de las flotas pesqueras exteriores y por el que se deroga el Reglamento (CE) 1006/2008 del Consejo, Ponente: Linnéa Engström, A8-0377/2016, 9-12-2016; así como, Parlamento Europeo, Resolución legislativa del Parlamento Europeo sobre la propuesta de Reglamento del Parlamento Europeo y del Consejo sobre la gestión sostenible de las flotas pesqueras exteriores y por el que se deroga el Reglamento (CE) 1006/2008 del Consejo, Procedimiento legislativo ordinario: primera lectura, P8_TA(2017)0015, 2-2-2017.

39 Parlamento Europeo, Provisional Agreement Resulting from Interinstitutional Negotiations, PE607.818V01-00, 26-6-2017. La primera lectura del Consejo recogió los términos del acuerdo provisional alcanzado, véase Consejo, Position of the Council at first reading with a view to the adoption of a Regulation of the European Parliament and of the Council on the sustainable management of external fishing fleets, and repealing Council Regulation (EC) 1006/2008, 17-10-2017, 11382/2/17 REV 2. Y, finalmente, la segunda lectura parlamentaria de la propuesta aprobó, sin enmienda alguna, los términos de la propuesta adoptados en primera lectura por el Consejo, Parlamento Europeo, Resolución legislativa del Parlamento Europeo, de 12-12-2017, respecto de la posición del Consejo en primera lectura con vistas a la adopción del Reglamento del Parlamento Europeo y del Consejo sobre la gestión sostenible de las 
sobre las solicitudes de reingreso al registro comunitario ha de proyectarse sobre la actividad desarrollada durante los últimos cinco años — como exigía la Comisión - por los buques que han sido objeto de reabanderamiento (art. 6). También ha sido meritorio el consenso alcanzado respecto al art. 5 que, durante el procedimiento, había levantado suspicacias entre los armadores de la flota de altura que consideraban que la propuesta inicial creaba «una doble penalidad ya que el buque y el capitán del buque estarían sujetos, no sólo a las sanciones previstas en los Reglamentos de control e INDNR, sino que, además, no podrían solicitar una autorización si, durante los doce meses anteriores, hubieran cometido una infracción grave, lo que se traduciría en un año de paralización ${ }^{40}$. El texto aprobado por los colegisladores comunitarios elimina dicho riesgo, al suprimir el art. 5.1.d que figuraba en el borrador original de la Comisión, que había sido rechazado por el Comité de Pesca del Parlamento y, posteriormente, reintroducido durante la votación del Pleno en primera lectura.

Este complejo proceso de negociación del Reglamento sobre la gestión sostenible de las flotas pesqueras exteriores ha propiciado una reflexión global sobre el papel que debe desempeñar la UE en el control de la actividad de la flota de aguas distantes. De forma lógica, en el transcurso de este debate ha resultado inevitable que el legislador tropezase con la invisibilizada problemática de los acuerdos durmientes. Si bien en la iniciativa de la Comisión no se realizaba mención alguna a este tema, en el trámite parlamentario se introdujo la enmienda núm. 48, relativa al art. 17 de la propuesta, con el objetivo de formular una solución al atolladero jurídico que suscita la eficacia de la cláusula de exclusividad sobre dichos convenios. El texto de la enmienda indicaba que «un Estado miembro del pabellón podrá expedir una autorización de pesca para las actividades pesqueras llevadas a cabo en aguas de terceros países siempre que el protocolo de un acuerdo de colaboración de pesca sostenible aplicable a dichas aguas no haya estado en vigor en el tercer país en cuestión

flotas pesqueras exteriores y por el que se deroga el Reglamento (CE) 1006/2008 del Consejo. Segunda lectura, P8_TA-PROV(2017)0475.

40 EUROPECHE, comunicado de prensa, 3-2-2017. En particular, el problema se planteaba respecto al art. 5.1.d de la propuesta original de la Comisión, que señalaba que el operador de la pesca y el buque pesquero solo podía solicitar una autorización de pesca si no había sido objeto de una sanción por una infracción grave durante los doce meses anteriores, lo que ciertamente añadía una sanción suplementaria a la ya recibida por el armador por la comisión de la infracción prevista en el Reglamento INDNR. $\mathrm{Al}$ respecto de dicha posición véase también el informe «Sanciones por partida doble» recogido en Pesca Internacional, ním. 181, marzo de 2017, pp. 4-6. 
durante al menos los tres años precedentes ${ }^{41}$; es decir, la enmienda permitía hacer compatibles las licencias privadas y los acuerdos de flete con los ACPS en los supuestos de tratados que hubiesen permanecido durmientes durante tres años.

La ponente parlamentaria se opuso vehementemente a la enmienda y señaló en el debate plenario que esta abriría la puerta al acceso de la flota comunitaria a la ZEE de los ribereños bajo estándares de sostenibilidad más débiles que los de los contenidos en los ACPS, vulnerando de este modo el objetivo de la PPC ${ }^{42}$. Resulta difícil poder compartir dicho enfoque cuando la meta expresa del Reglamento (UE) 2017/2403 es someter los acuerdos privados a un nivel de control análogo al existente para los acuerdos públicos. En el mismo debate, el comisario de Pesca se limitó a indicar que, aunque simpatizaba con la propuesta, no la apoyaba por motivos legales, anticipando la respuesta oficial proporcionada por la Comisión Europea durante el iter legislativo (en el momento de valorar la primera lectura realizada por el Parlamento y de remitirla al Consejo) que se limitó a repetir, de manera un tanto tautológica, que la enmienda concebida para resolver los efectos indeseados de las cláusulas de exclusividad no resultaba aceptable "porque sería contraria a los acuerdos que incluyen una cláusula de exclusividad ${ }^{43}$.

41 En la justificación de la enmienda se hacía referencia al carácter estructural e irresuelto de la cuestión al señalar que «given that the issue of some dormant SFPAs with unimplemented Protocols has not been solved, the EU should offer a solution to allow for direct fishing authorisations in these cases under certain conditions», Parlamento Europeo, Amendments 001-076 by the Committee on Fisheries, Sustainable management of external fishing fleets, Proposal for a regulation (COM(2015)0636 - C80393/2015 - 2015/0289(COD)), 25-1-2017.

42 En el debate mantenido en sede parlamentaria, Linnéa Engström (Greens/EFA, Sweden) señaló que 《if vessels are allowed after three years of a dormant agreement to set up their own terms with a third country, they will be able to ignore those very provisions in the CFP that have served to improve EU standards. They could negotiate weaker terms despite the existence of a bilateral agreement, and that is not maintaining the high standards of the EU. It is serving to undermine them. So I urge you all to keep up the good standards of EU external fishing and to refer to, and vote against, Amendment 48》, Parlamento Europeo, Acta de la sesión del 1-2-2017, Bruselas, P8_PV(2017)02-01, PE 599.240-3.

43 Comisión Europea, Ordinary legislative procedure - First reading, European Parliament legislative resolution of 2 February 2017 on the proposal for a Regulation of the European Parliament and of the Council on the sustainable management of external fishing fleets, repealing Council Regulation (EC) 1006/2008, 2015/0289 (COD), 14-3-2017. 
La pequeña rendija de oportunidad, más que ventana, abierta por la enmienda 48 para la resolución del entuerto causado por el efecto de las cláusulas de exclusividad sobre los acuerdos durmientes quedó clausurada, por lo tanto, de una forma bastante abrupta. A pesar del desenlace, la enmienda resultaba razonable, congruente con la visión holística del control de la actividad de la flota en aguas extracomunitarias que le corresponde asumir a la UE y, también, coherente con los intereses del sector ${ }^{44}$. Sin embargo, la enmienda 48 naufragó en el procedimiento legislativo y la única referencia a los acuerdos pesqueros sin protocolo que encontramos en el texto del Reglamento (UE) 2017/2403 es un nuevo considerando núm. 19, que ha sido añadido al inicio de la norma y en el que se recuerda que «los buques pesqueros de la Unión no están autorizados a faenar en aguas bajo la jurisdicción o soberanía de terceros países con los que la Unión tenga un acuerdo pero no un protocolo en vigor», para señalar a continuación que «en el caso de un acuerdo de este tipo, cuando durante al menos tres años no haya en vigor ningún protocolo, la Comisión debe examinar las causas de la situación y tomar las medidas apropiadas, que pueden incluir la propuesta de negociar un nuevo protocolo» ${ }^{45}$. El impreciso enunciado que el legislador comunitario ha optado por dar a la disposición deja espacio para que nos preguntemos si esta referencia resultará suficiente para dar respuesta al postergado problema de los acuerdos durmientes.

\section{CONCLUSIONES}

En principio, no parece que la fórmula incluida en el nuevo Reglamento sobre la gestión sostenible de las flotas exteriores solucione, de manera definitiva, el callejón sin salida al que aboca en términos jurídicos la cláusula de exclusividad de los acuerdos durmientes. Para empezar, porque el legislador ha preferido insertar una referencia indirecta a la cuestión entre los numerosos considerandos iniciales del Reglamento (UE) 2017/2403, en vez de dedicarle un artículo regulatorio, como había sido solicitado en el trámite de enmiendas. Pero, sobre todo, porque la solución prevista —incluso aunque supone un avance sobre la situación actual - no solventa de manera automática el problema, sino que se limita a incorporar una imprecisa obligación

44 LDAC, Recomendaciones para garantizar un nuevo Reglamento de Autorizaciones de Pesca (FAR) sólido y consistente para la gestión sostenible de la flota pesquera exterior, 4-11-2016, R-10-16/GT5.

45 Reglamento (UE) 2017/2403..., loc.cit., supra. 
de comportamiento a cargo de la Comisión Europea que, cuando se dé la circunstancia de que un ACPS permanezca sin protocolo tres años, deberá examinar la situación y adoptar las medidas adecuadas. La redacción dada a este considerando resulta demasiado abierta, sobre todo si tenemos en cuenta la contumaz inoperatividad de la Comisión frente a este problema, puesto que no señala qué conducta debe ser adoptada si no resulta posible negociar un nuevo protocolo; en ese sentido, hubiese resultado preferible establecer con claridad que si, tras el transcurso del plazo de tres años de latencia del acuerdo, no ha sido suscrito un nuevo protocolo de ejecución, debería permitirse - de forma mecánica - la celebración de acuerdos privados o procederse a consumar inmediatamente la denuncia del acuerdo ${ }^{46}$.

Aunque en ocasiones parece perderse de vista, las cláusulas de exclusividad no constituyen un fin en sí mismo de la práctica convencional pesquera de la UE, sino que su funcionalidad radica en permitir que se produzca un control efectivo de la actividad de la flota, a fin de garantizar que los navíos comunitarios que faenen en aguas de los Estados socios se encuentren sometidos al enfoque responsable y a los principios de gobernanza que impulsan los ACPS (Panossian, 2015: 3). Por coherencia lógica, al establecerse, tras la entrada en vigor del Reglamento (UE) 2017/2403, un control sobre los acuerdos privados y de flete que resulte equiparable al que existe sobre las licencias expedidas en virtud de los acuerdos pesqueros, desaparece el motivo para mantener vigentes aquellos ACPS que se encuentran carentes de protocolo y que, de hecho, han bloqueado durante años cualquier otra vía de ingreso a las ZEE de los Estados ribereños. En realidad, resultaría también razonable considerar, de cara al futuro, una eventual reforma de la técnica jurídica relativa a la inclusión de las cláusulas de exclusividad en tratados de pesca, puesto que, a fin de evitar disfuncionalidades, estas disposiciones encuentran un mejor encaje normativo en los protocolos de ejecución de los tratados (impidiendo que se produzca el acceso simultáneo de la flota comunitaria a las ZEE de países terceros mediante licencias privadas y públicas derivadas de ACPS) que en los propios acuerdos marco, habida cuenta de

46 En este sentido, la reciente (e inhabitual, véase supra., nota 24) iniciativa de la Comisión Europea de solicitar la denuncia del ACPS con Comoras, tras la calificación de este Estado como no cooperante en materia de pesca INDNR, constituye un referente de buena práctica que debiera ser tomada en consideración como precedente para la actuación futura, al constituir una respuesta ágil (atributo este del que no ha hecho gala la Comisión en el caso de los acuerdos durmientes) por parte del Ejecutivo comunitario y plenamente coherente con el derecho de la UE vigente en este ámbito; véase Comisión Europea, Propuesta de Decisión del Consejo por la que se denuncia..., loc. cit., supra. 
que, cuando figuran en los segundos, su efecto termina siendo, si no existe protocolo en vigor, el de impedir cualquier otra fórmula alternativa de pesca; lo que se compadece mal con el objetivo fundacional y básico de la PPC de «generar beneficios económicos, sociales y de empleo, y de contribuir a la disponibilidad de productos alimenticios ${ }^{47}$.

Los ejemplos más extremos de esta situación se suscitan en los casos de Gambia y Guinea Ecuatorial. En los dos supuestos los acuerdos pesqueros llevan durmiendo más de quince años y en las ZEE de ambos Estados ha sido verificada la existencia de prácticas pesqueras por parte de la flota comunitaria en contravención de las cláusulas de exclusividad. Aún más, por relación a ambos escenarios ha sido identificado un interés concreto de la flota en continuar desarrollando actividades extractivas ${ }^{48}$. Sin embargo, la relación bilateral existente entre dichos Estados y la UE se encuentra trabada por la presencia de obstáculos políticos significativos, por lo que las perspectivas reales de suscripción de nuevos protocolos, después de casi dos décadas, continúan siendo remotas.

Ante esta tesitura cabe preguntarse de qué opciones podrían servirse los operadores pesqueros comunitarios para impulsar la reacción institucional de la UE, si la Comisión Europea decidiese continuar instalada — tras la entrada en vigor del Reglamento (UE) 2017/2403 — en la pasividad que ha caracterizado su aproximación a la problemática de los acuerdos durmientes. A nuestro juicio, en primer término y como prerrequisito de cualquier otra actuación subsiguiente, debería realizarse una estimación solvente del coste económico que tiene para el sector privado la pervivencia de la cláusula de exclusividad en los acuerdos pesqueros sin protocolo; a través de sus organizaciones representativas, los operadores con intereses económicos en las aguas de los Estados ribereños afectados deben determinar el alcance del daño patrimonial sufrido por la pérdida de oportunidades de pesca originada por acuerdos durmientes de longa data como los de Guinea Ecuatorial o Gambia a fin de poder hacer efectivos tanto un requerimiento de actuación a las instituciones europeas competentes como una eventual reparación.

47 Art. 2 del Reglamento (UE) 1380/2013, loc.cit.

48 En el caso de Guinea Ecuatorial se ha señalado que «para las flotas de la UE, la zona de pesca de Guinea Ecuatorial es clave para los atuneros cerqueros. La reciente suspensión, de manera indefinida, de las posibilidades de pesca negociadas al amparo del ACPS con Gabón hace más necesario si cabe el acceso a la zona para no comprometer la estrategia pesquera regional para este segmento de la flota», COFREPECHE et al. (2016: 21). 
En segundo lugar, resultaría necesario agotar las posibilidades de control político que existen sobre la iniciativa legislativa de la Comisión. Han sido planteadas ya algunas preguntas parlamentarias individuales respecto a este asunto ${ }^{49}$, pero cabe recordar que, en virtud del art. 225 TFUE, el Parlamento Europeo tiene la facultad de solicitar a la Comisión que presente «las propuestas oportunas sobre cualquier asunto que requiera la elaboración de un acto de la Unión para la aplicación de los Tratados», como ocurre en el caso de la denuncia de un tratado de pesca suscrito con un tercer Estado; estando obligada la Comisión, si no presenta iniciativa alguna, a comunicar sus razones para no hacerlo. El Consejo dispone de una facultad equivalente en virtud del art. 241 TFUE y, de no observarse un desbloqueo efectivo de la situación por parte de la Comisión, los Estados más directamente afectados por la inactividad del Ejecutivo (como es el caso de España) podrían plantearse instar una respuesta a través de esta vía.

Por último, restaría para los afectados la opción, que habría no obstante que contemplar con considerable escepticismo, de promover un control jurisdiccional sobre la inactividad de la Comisión Europea a la hora de impulsar la denuncia del acuerdo, a través del recurso previsto en el art. 265 TFUE. A pesar de que discutir la conducta omisiva de la Comisión en este asunto parece complicado a la luz de los hechos — sobre todo si tenemos en mente los acuerdos que han permanecido profundamente dormidos durante décadas-, la vía jurisdiccional se encuentra plagada de obstáculos que dificultarían el avance del proceso. En primer término, debido a la restrictiva legitimación activa de los particulares perjudicados por la inacción de las instituciones comunitarias para plantear el recurso y la consabida inexistencia de vías indirectas para atacar dicha conducta omisiva, a diferencia de lo que ocurre con la relación que se establece entre el recurso de anulación y la cuestión prejudicial de validez o la excepción de legalidad. En segundo lugar, porque incluso aunque el recurso llegase a ser interpuesto por un demandante privilegiado como un Estado (España) o bien una institución (PE o Consejo), habría que contar con la tradicional renuencia que ha demostrado el TJUE a la hora de ejercitar un control jurisdiccional en el terreno de la producción normativa cuando existe, como ocurre en este caso, un cierto margen de apreciación en manos del poder decisorio europeo (Alonso García, 2000). Y, en último término, por

49 Preguntas parlamentarias con solicitud de respuesta escrita a la Comisión formuladas por Francisco José Millán Mon (PPE), 28-1-2016, E-000714-16; y Renata Briano (S\&D), 29-9-2016, E-007228-16, con respuestas (en absoluto clarificadoras sobre el fondo del asunto, conviene anotar) proporcionadas por el comisario Vella, en nombre de la Comisión Europea, respectivamente de 8-4-2016 y 25-11-2016. 
la propia ineficiencia del recurso por omisión dentro del sistema jurisdiccional comunitario (Daukšien y Budnikas, 2014), que puede ser bloqueado por la institución requerida mediante un simple rechazo expreso de la solicitud de actuación.

\section{Bibliografía}

Alonso García, R. (2000). Actividad judicial v. inactividad normativa (El Tribunal de Justicia de las Comunidades Europeas frente al déficit normativo de las Instituciones y de los Estados miembros). Revista de Administración Pública, 151, $77-132$.

COFREPECHE, NFDS, MRAG y POSEIDON (2015). Ex post and ex ante evaluation of the protocol to the Fisheries Partnership Agreement between the EU and the Republic of Mauritius (Framework contract MARE/2011/01-Lot 3, specific contract 16). Bruselas.

COFREPECHE, NFDS, POSEIDON y MRAG (2013a). Évaluation prospective de l.opportunité diun accord de partenariat dans le secteur de la pêche entre l. Union européenne et la République du Sénégal (Sous le Contrat cadre MARE/2011/01Lot 3, contrat spécifique 5). Bruselas.

COFREPECHE, POSEIDON, MRAG y NFDS (2013b). Revue des pêcheries thonières dans l'océan Atlantique Est (Contrat cadre MARE/2011/01-Lot 3, contrat spécifique 5). Bruselas.

- (2012). Évaluation ex-post du protocole de l'accord de partenariat dans le domaine de la pêche entre l,Union européenne et la Côte-dilvoire (Contrat cadre MARE/2011/01-Lot 3, contrat spécifique 2). Bruselas.

COFREPECHE, POSEIDON, NFDS y MRAG (2016). Évaluation prospective d'un protocole à un accord de partenariat dans le domaine de la pêche durable entre l,Union européenne et la République de Guinée équatoriale (Contrat cadre MARE/2011/01-Lot 3, contrat spécifique 18). Bruselas.

Confederación Española de Pesca (CEPESCA). (2017). Informe del sector pesquero español 2017. Disponible en: https://goo.gl/8k48ao.

Daukšienė, I. y Budnikas, A. (2014). Has the action for failure to act in the European Union lost its purpose? Baltic Journal of Law \& Politics, 7 (2), 209-226. Disponible en: https://goo.gl/H2uaVq.

EJF, OCEANA, PEW y WWF (2016). European vessels fishing under the radar.

García Andrade, P. (2016). La opinión consultiva del TIDM de 2 de abril de 2015: cuestiones de representación exterior y responsabilidad internacional de la Unión Europea. En J. M. Sobrino (dir.). La toma de decisiones en el ámbito marítimo: su repercusión en la cooperación internacional y en la situación de las gentes del mar (pp. 133-153). Albacete: Bomarzo.

Hudson, A. (2007). Case Study: The Fisheries Partnership Agreements. London: Overseas Development Institute. 
Jablonicky, C., McCauley, D., Kroodsma, D., Boerder, K. y Dunn, D. (2016). Satellite tracking to monitor area-based management tools \& identify governance gaps in fisheries beyond national jurisdiction. Nereus Scientific \& Technical Briefs on $A B N J$ series. Disponible en: https://goo.gl/zzD9oK.

Jorge Urbina, J. (2017). Fortalecimiento de los controles sobre los operadores involucrados en actividades relacionadas con la pesca ilegal, no declarada y no reglamentada. En J. A. Pueyo y J. Jorge Urbina (coords.). La reforma de la gobernanza pesquera internacional y europea (pp. 171-217). Thomson Reuters-Aranzadi.

Long Distance Advisory Council (LDAC). (2015). Conference on External Dimension of the CFP, Las Palmas de Gran Canaria, 16-17 September. Recommendations.

Ministerio de Agricultura, Pesca, Alimentación y Medio Ambiente (MAGRAMA). (2017). Censo de la flota pesquera operativa 2017. Disponible en: https://goo.gl/ Yr4THn.

Mulazzani, L. y Malorgio, G. (2015). Is there coherence in the European Union's strategy to guarantee the supply of fish products from abroad? Marine Policy, 52, 1-10. Disponible en: https://doi.org/10.1016/j.marpol.2014.10.018.

NFDS, POSEIDON, COFREPECHE y MRAG (2014). Ex ante evaluation of a possible future fisheries partnership agreement and protocol between the European Union and the United Republic of Tanzania (Framework contract MARE/2011/01-Lot 3, specific contract 7). Bruselas.

Oanta, G. A. (2016). Tres sentencias claves para la delimitación del contorno jurídico de las competencias convencionales de la Unión Europea en el ámbito pesquero. Revista de Derecho Comunitario Europeo, 53, 201-231. Disponible en: https://doi.org/10.18042/cepc/rdce.53.05.

OCEANA (2017). Fishing the Boundaries of Law. How the exclusivity clause in EU Fisheries Agreements was Undermined.

Oceanic Développement y Megapesca Lda (2011). Ex ante evaluation of existing conditions in the fisheries sector in Mauritius with a view to concluding a new fisheries partnership agreement and protocol (Framework contract FISH/2006/20-Convention spécifique 34). Bruselas.

- (2014a). Ex-post and ex-ante evaluations of the protocol to the Fisheries Partnership Agreement between the EU and the Republic of Mozambique (Framework contract FISH 2011/01-Lot 3, specific contract 11). Bruselas.

- (2014b). Ex-post and ex-ante evaluations of the protocol to the Fisheries Partnership Agreement between the EU and Kiribati (Framework contract FISH 2011/01Lot 3, specific contract 12). Bruselas.

Panossian, A. (2015). Accords de Partenariat de Pêche Durable: Une nécessaire harmonisation de la portée de la clause d'exclusivité et de son interprétation. Coalition for Fair Fisheries Arrangements.

Penas Lado, E. (2016). The Common Fisheries Policy. Wiley Blackwell. Disponible en: https://doi.org/10.1002/9781119085676. 
Popescu, I. (2015). Beyond the European Seas. The External Dimension of the Common Fisheries Policy. European Parliamentary Research Service. Disponible en: https://goo.gl/8r8Y2s.

- (2016). Expanding the network of EU tuna fisheries agreements. European Parliamentary Research Service. Disponible en: https:/goo.gl/88W8et.

POSEIDON, MRAG, COFREPECHE y NFDS (2012). Ex post evaluation of the current Protocol to the Fisheries Partnership Agreement between the European Union and Republic of Kiribati, and ex ante evaluation including an analysis of the impact (Framework contract MARE/2011/01-Lot 3, specific contract 01). Bruselas.

- (2013). Review of tuna fisheries in the Pacific Ocean (Framework contract MARE/2011/01-Lot 3, specific contract 6). Bruselas.

- (2014a). Ex ante evaluation of a possible future fisheries partnership agreement and protocol between the European Union and Kenya (Framework contract MARE/2011/01-Lot 3, specific contract 7). Bruselas.

POSEIDON, MRAG, NFDS y COFREPECHE (2014b). Review of tuna fisheries in the western Indian Ocean (Framework contract MARE/2011/01-Lot 3, specific contract 7). Bruselas.

Robards, M., Silber, G., Adams, J., Arroyo, J., Lorenzini, D., Schwehr, K. y Amos, J. (2016). Conservation science and policy applications of the marine vessel Automatic Identification System (AIS) - a review. Bulletin of Marine Science, 92 (1), 75-103. Disponible en: https://doi.org/10.5343/bms.2015.1034.

Sobrino Heredia, J. M. y Oanta, G. A. (2015). The Sustainable Fisheries Partnership Agreements of the European Union and the Objectives of the Common Fisheries Policy: Fisheries and/or Development? Spanish Yearbook of International Law, 19, 61-85. Disponible en: https://doi.org/10.17103/sybil.19.04.

STECF European Commission, Scientific, Technical and Economic Committee for Fisheries (2017). The 2017 Annual Economic Report on the EU Fishing Fleet. Luxembourg: Publications Office of the European Union. Disponible en: https://goo.gl/dbSQMF.

Teijo García, C. (2016). Los Acuerdos de Cooperación para la Pesca Sostenible de la UE en el marco de la reforma de la dimensión exterior de la Política Pesquera Común. En J. A. Pueyo y J. Jorge (coords.). La gobernanza maritima europea: retos planteados por la reforma de la Politica Pesquera Común (pp. 235-264). Thomson Reuters-Aranzadi.

Tribunal de Cuentas Europeo (2015). ¿Gestiona la Comisión correctamente los acuerdos de colaboración en el sector pesquero? Informe especial, no 11 . Luxemburgo: Oficina de Publicaciones de la Unión Europea.

Walmsley, S. F., Barnes, C. T., Payne, I. A. y Howard, C. A. (2007). Comparative Study of the Impact of Fisheries Partnership Agreements. Technical Report. Londres: MRAG. 